\title{
ANÁLISIS, DISEÑO Y EVALUACIÓN SÍSMICA DE EDIFICIOS ALTOS DE CONSTRUCCIÓN COMPUESTA Y CON ARRIOSTRAMIENTOS DE PANDEO RESTRINGIDO
}

ANALYSIS, DESIGN AND SEISMIC EVALUATION OF TALL BUILDINGS OF
MIXED CONSTRUCTION AND WITH RESTRICTED BUCKLING BRACINGS

PEDRO ROJAS C. ${ }^{1}$, JOSÉ BARROS $C^{2}{ }^{2}$, MARIO AGUAGUIÑA M. ${ }^{3}$, RICARDO HERRERA M. ${ }^{4}$

RESUMEN

Este artículo forma parte de un plan de investigación que busca proporcionar a la industria ecuatoriana de la construcción en acero alternativas de sistemas estructurales para edificios altos y de mediana altura, diferentes a los de acero y hormigón armado convencionales usados actualmente en Guayaquil. Los Pórticos Resistentes a Momento Compuestos (PRMC) con columnas CFT (CFT, Concrete-Filled Steel Tube Column, por su nombre en inglés) y los Pórticos con Arriostramientos de Pandeo Restringido (PAPR) son los sistemas estructurales que se presentan en este estudio. Se realiza primero una revisión de los aspectos más importantes relacionados al análisis y diseño de un edificio prototipo de 24 pisos de altura en el que los sistemas PRMC y PAPR constituyen el Sistema Resistente a Cargas Sísmicas. Este diseño fue realizado en base a las disposiciones de diseño sísmico de códigos Norteamericanos y recomendaciones de investigaciones previas relacionadas a estos sistemas. Luego se, describe el desarrollo del modelo no-lineal de los PRMC y PAPR y la calibración realizada para cada uno de los componentes estructurales (vigas, columnas CFT, conexiones viga- columna y arriostramientos de pandeo restringido) de estos sistemas. Finalmente, se presentan los resultados de los análisis estático (pushover) y dinámico no lineales correspondientes al edificio prototipo. Los cuales indican que ambos sistemas tienen un adecuado desempeño sísmico y consecuentemente constituyen alternativas interesantes para la construcción de edificios altos y de mediana altura en nuestro país.

PALABRAS CLAVE: diseño sísmico, evaluación sísmica, sistema estructural, pórticos resistentes a momento compuestos, pórticos con arriostramientos de pandeo restringido

\section{ABSTRACT}

This article is part of a research plan that seeks to provide the Ecuadorian steel construction industry with alternative structural systems for tall and medium height buildings, different from those of conventional steel and concrete currently used in Guayaquil. Compound Moment Resistant Frames (PRMC) with CFT columns (concrete-filled steel tube column), and Restricted Buckling Bracings (PAPR) are the structural systems presented in this study. First, a review of the most important aspects related to the analysis and design of a 24-story high-rise building in which the PRMC and PAPR systems constitute the Seismic Load Resistance System. This design was made based on seismic design provisions of North American codes and recommendations of previous investigations related to these systems. Then, it describes the development of the non-linear model of the PRMC and PAPR and the calibration performed for each of the structural components (beams, CFT columns, beam-column connections and constrained buckling bracing) of these systems. Finally, the results of the non-linear static and pushover analysis of the prototype building are presented. These indicate that both systems have an adequate seismic performance and consequently are interesting alternatives for the construction of high and medium height buildings in our country.

KEYWORDS: seismic design, seismic evaluation, structural system, momentresistant composite frames, restricted bracing frames 


\section{INTRODUCCIÓN}

Este artículo presenta la etapa inicial de un programa de investigación ambicioso que busca estudiar analítica y experimentalmente dos sistemas estructurales comúnmente usados en edificios de mediana altura y altos en otros países. Los Pórticos Resistentes a Momento Compuestos (PRMC) con columnas CFT así como los Pórticos con Arriostramientos de Pandeo Restringido (PAPR) son los sistemas estructurales propuestos en este programa como alternativa a los sistemas de construcción en acero y en hormigón armado convencionales que se emplean en Ecuador. El plan de investigación se ha llevado a cabo en cuatro fases: (I) estudio analítico sobre análisis, diseño y comportamiento de los sistemas PRMC y PAPR; (II) ensayo y calificación de conexiones a momento viga-columna CFT, incluyendo especímenes en forma de " $\mathrm{T}$ " y especímenes cruciformes; (III) ensayo y calificación de arriostramientos de pandeo restringido (APR), incluyendo especímenes de APR individual y especímenes de APR en subestructura; y (IV) desarrollo de disposiciones para el diseño y construcción de edificios compuestos por PRMC y PAPR en Ecuador. La figura 1 presenta las distintas fases del plan de investigación planteado.

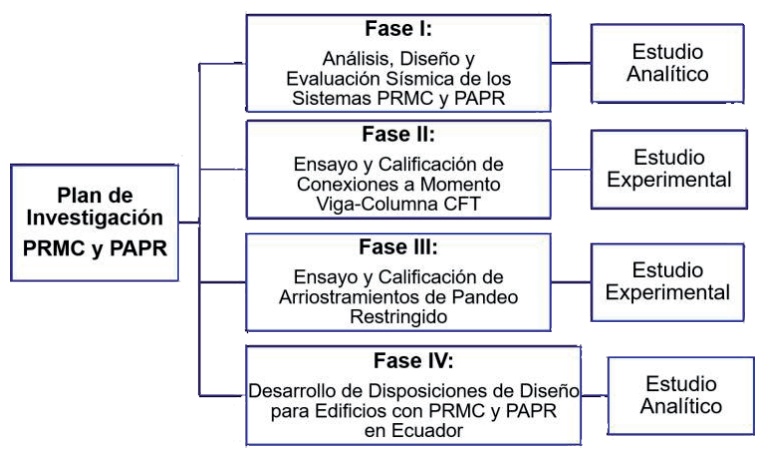

Figura 1. Plan de Investigación para el estudio de los sistemas estructurales PRMC y PAPR.

El artículo destaca los resultados parciales de la primera fase de este plan de investigación. En este trabajo se exponen los criterios de análisis y diseño de los sistemas estructurales PRMC y PAPR. Estos criterios fueron aplicados en el diseño de un edificio prototipo de 24 pisos de altura cuyo Sistema Resistente a Cargas Sísmicas está conformado por PRMC y PAPR. Adicionalmente, se presentan los resultados de los análisis estático (pushover) y dinámico (tiempo-historia) no lineales realizados al edificio prototipo.

\section{INVESTIGACIÓN PREVIA}

El hormigón armado ha sido y es el material más usado en la construcción de edificios en la ciudad de Guayaquil, Ecuador. Los Pórticos Resistentes a Momento (PRM) es el sistema estructural más común para la construcción de edificios de hormigón armado. Sin embargo, la construcción de edificios de acero estructural se ha incrementado durante los últimos 15 años en la ciudad de Guayaquil. La mayoría de los edificios de acero construidos en este período corresponden a estructuras de pocos pisos compuestas principalmente por PRM.

Una investigación fue llevada a cabo en el 2009 con el fin de estudiar el estado del arte del diseño y construcción de los edificios de acero ubicados en la ciudad de Guayaquil (Cassagne, 2009). Los resultados de esta investigación revelaron que algunos de los edificios construidos antes del 2008 presentaban algunas deficiencias relacionadas al diseño, detalles constructivos (e.g., uso de conexiones no precalificadas, soldadura inadecuada), mano de obra e inspección. Adicionalmente, algunos de estos edificios fueron diseñados sin considerar los efectos de la carga sísmica debido a que versiones previas del Código Ecuatoriano de la Construcción (CEC) no incluían requerimientos sísmicos para estructuras de acero. Como consecuencia, estas estructuras podrían presentar un comportamiento no dúctil ante la ocurrencia de un evento sísmico severo (Cassagne, 2009).

La Norma Ecuatoriana de la Construcción (NEC-15), código que rige el diseño y construcción de las estructuras en el Ecuador, sí incluye disposiciones de diseño sísmico y requerimientos constructivos para estructuras de acero. Estas disposiciones forman parte del capítulo NEC-SE-AC - Estructuras de Acero. No obstante, la NEC-15 proporciona solamente tres alternativas para sistemas estructurales que pueden ser empleados como parte del Sistema Resistente a Cargas Sísmicas (SRCS) de edificios de acero estructural. Estos sistemas son: (1) Pórticos Resistentes a Momento Especiales; (2) Pórticos Arriostrados Concéntricamente Especiales: y (3) Pórticos Arriostrados Excéntricamente Especiales. Los sistemas estructurales (2) y (3) casi no han sido aplicados en Ecuador. Las disposiciones de diseño de estos sistemas se basan fundamentalmente en códigos Norteamericanos. Las estructuras de construcción compuesta (i.e., PRMC) al igual que los PAPR no están contemplados en la NEC-15. Por otra parte, la mayoría de los edificios considerados como altos han empleado hormigón armado 
como material principal en su construcción y los autores no tienen constancia de edificios de acero que excedan los 10 pisos de altura en la ciudad de Guayaquil. Por esta razón, se propone realizar un estudio de los sistemas PRMC y PAPR para proporcionar a la industria de la construcción en acero local otras alternativas de sistemas estructurales para edificios de mayor altura que los que existen actualmente.

Los sistemas estructurales PRMC y PAPR han sido ampliamente estudiados en varios países, principalmente en Estados Unidos y Japón. Investigaciones analíticas y experimentales han demostrado que estos sistemas presentan un desempeño sísmico superior en comparación con pórticos resistentes a momento de hormigón armado o de acero, y pórticos arriostrados convencionales. (Herrera, 2005; Fahnestock, et al., 2006).

PÓRTICOS RESISTENTES A MOMENTO COMPUESTOS (PRMC) Los Pórticos Resistentes a Momento Compuestos (PRMC) con columnas CFT presentan ventajas tanto económicas como prácticas en comparación con pórticos a momento de hormigón armado y de acero convencionales. Las columnas CFT son miembros compuestos conformados por un perfil tubular de acero cuadrado, rectangular o circular relleno de hormigón (ver figura 2). Las columnas CFT poseen mayor rigidez lateral que miembros de acero y la interacción entre el acero y el hormigón resulta en miembros con mayor capacidad a carga axial y a flexión. Además, el tubo de acero proporciona un efecto de confinamiento al hormigón así como el pandeo local del tubo de acero es retardado por el efecto de restricción del relleno de hormigón. Las columnas CFT pueden optimizar la cantidad de acero y el uso de material contra fuegos puede ser reducido debido a la resistencia al fuego del hormigón. Adicionalmente, no se necesita encofrado, lo cual resulta en un ahorro de mano de obra y reducción de los tiempos de construcción. (Morino \& Tsuda, 2003; Muhummud, 2003).
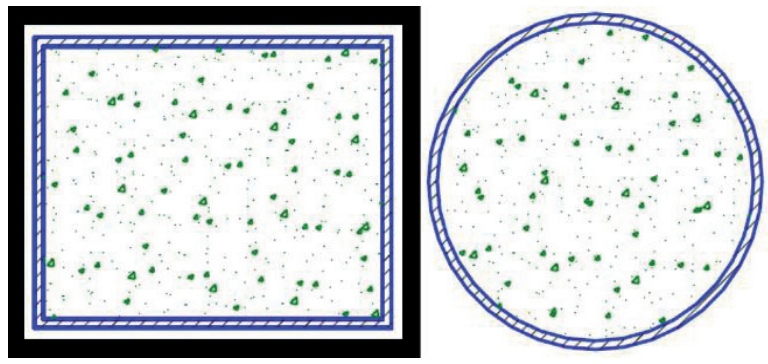

Figura 2. Columnas CFT: Perfiles tubulares de acero rellenos de hormigón (Crisafulli, 2013).

\section{PÓRTICOS CON ARRIOSTRAMIENTOS DE PANDEO} RESTRINGIDO (PAPR)

Los Pórticos con Arriostramientos de Pandeo Restringido (PAPR) son un tipo especial de pórtico arriostrado concéntricamente que incorpora arriostramientos de pandeo restringido (APR). Un APR consiste en un elemento de acero esbelto, denominado núcleo de acero, el cual resiste la carga axial total del arriostramiento, y un mecanismo de restricción que elimina el modo de falla por pandeo del núcleo cuando éste se encuentra bajo fuerzas de compresión altas. La figura 3 muestra un esquema de un APR y sus diferentes componentes. Un APR es capaz de alcanzar la fluencia bajo fuerzas de tensión y de compresión sin presentar degradación de resistencia ni de rigidez importante. Consecuentemente, los PAPR presentan una respuesta histerética estable, ductilidad significativa y gran capacidad de disipación de energía cuando están sujetos a carga cíclica. Además, los APR tienen un desempeño sísmico superior en comparación con arriostramientos convencionales. (Sabelli \& López, 2004; Hussain, et al., 2006)

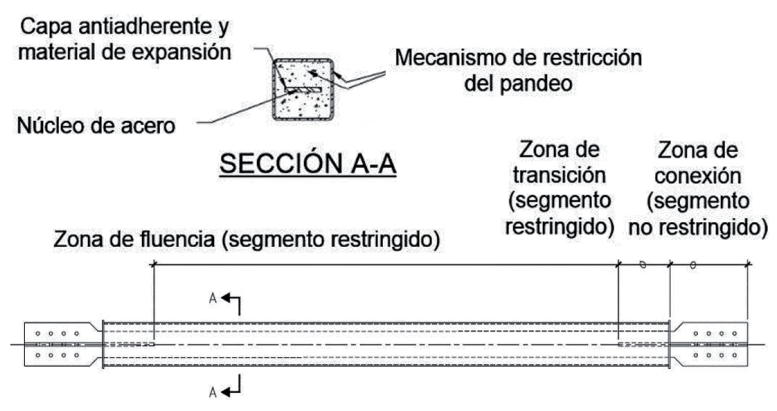

Figura 3. Componentes de un APR (López \& Sabelli, 2004).

\section{DISEÑO DEL EDIFICIO PROTOTIPO}

La primera fase del plan de investigación es un estudio analítico (ver figura 1) y supone la base de los futuros estudios experimentales. El objetivo principal de esta fase es el de estudiar los criterios de análisis y diseño de los sistemas estructurales PRMC y PAPR, incluyendo aspectos constructivos. Para alcanzar este objetivo, en esta fase se han planteado cuatro actividades principales: (1) revisión de las investigaciones más relevantes así como las disposiciones de análisis y diseño establecidas en códigos Norteamericanos acerca de estos sistemas (PRMC y PAPR); (2) aplicación de estos criterios y recomendaciones al diseño de un edificio prototipo de 24 pisos de altura compuesto por PRMC y PAPR; (3) evaluación del desempeño sísmico del edificio prototipo diseñado; y (4) desarrollo de recomendaciones para los futuros estudios ex- 
perimentales. Este estudio considera el estado del arte y la práctica del diseño y construcción de edificios de acero en el país. El edificio prototipo planteado como objeto de estudio podría ser considerado como una estructura alta en la ciudad de Guayaquil. En este artículo se presentan los resultados de las actividades 1, 2 y 3 .

Para alcanzar el objetivo principal de la Fase I del plan de Investigación se diseñó un edificio prototipo compuesto por PRMC y PAPR usando las disposiciones sísmicas de códigos Norteamericanos y recomendaciones de investigaciones previas. Una planta típica del edificio prototipo se muestra en la figura 4. Vistas en elevación de pórticos tipo en las direcciones X y Y se presentan en la figura 5.

El edificio prototipo estudiado corresponde a un edificio de oficinas de 24 pisos de altura localizado sobre suelo rígido en la ciudad de Guayaquil, Ecuador, una zona catalogada como de alto riesgo sísmico según la NEC-15. El edificio tiene tres vanos de $9.00 \mathrm{~m}$ en la dirección $\mathrm{X}$; y dos vanos exteriores de $5.00 \mathrm{~m}$ más un vano central de $6.00 \mathrm{~m}$ en la dirección Y. Como se observa en la figura 5(b), los APR fueron dispuestos en una configuración de diagonal simple, de forma simétrica, en los vanos exteriores de los PAPR. El área en planta del edificio es de $432.00 \mathrm{~m}^{2}$. La altura del primer entrepiso es de $4.25 \mathrm{~m}$ mientras que la altura de los entrepisos restantes es de $3.25 \mathrm{~m}$, lo cual resulta en una altura total de $79.00 \mathrm{~m}$ medidos desde la planta baja.

El Sistema Resistente a Cargas Sísmicas (SRCS) del edificio prototipo está conformado por PRMC en la dirección X y una combinación de PRMC y PAPR en la dirección Y. Como se observa en la figura 4, en la dirección Y los pórticos exteriores son PAPR mientras que los pórticos interiores son PRMC. Contrario a la práctica constructiva Norteamericana, todos los pórticos del edificio (cuatro en cada dirección) fueron diseñados para resistir tanto cargas gravitacionales como los efectos de la carga sísmica. Las dimensiones en planta y en elevación del edificio son típicas en la ciudad de Guayaquil. El edificio no presenta irregularidades en planta o en elevación.

Para justificar el uso de PRMC en una dirección y una combinación de PRMC y PAPR en la otra, las dimensiones del edificio prototipo fueron establecidas de manera que la relación altura-lado (H/B), conocida como relación de esbeltez, sea alrededor de 3 (2.93) y 5 (4.94) en las direcciones X y $\mathrm{Y}$, respectivamente.

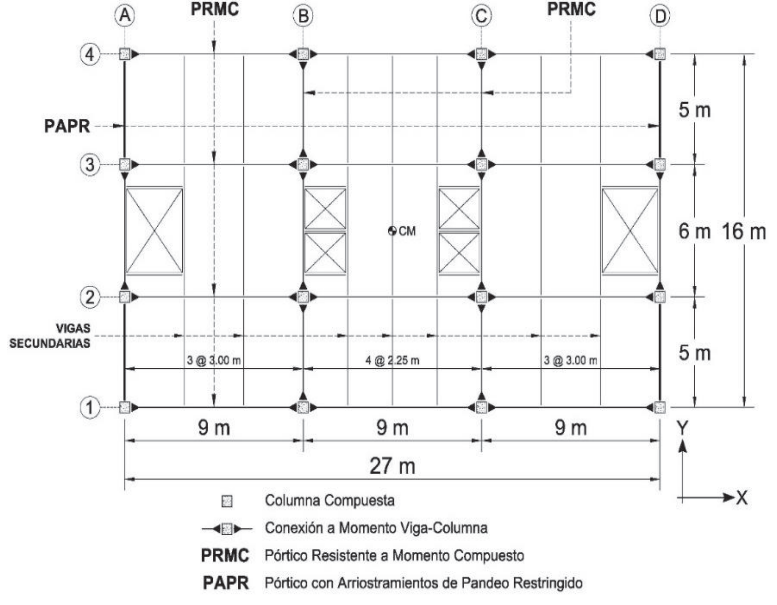

Figura 4. Planta del edificio prototipo.
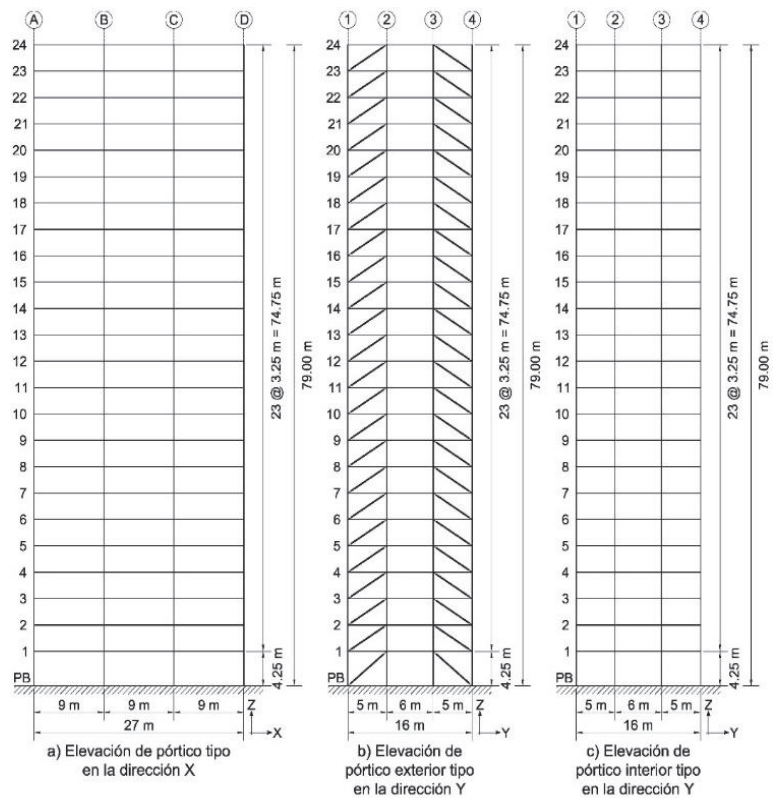

Figura 5. Elevaciones de pórticos tipo en las direcciones $X(P R M C)$ y $Y$ (PRMC-PAPR).

Todas las columnas del edificio prototipo son columnas CFT cuadradas armadas a base de placas soldadas. El material de las columnas es acero ASTM A992 $\left(\mathrm{Fy}=3500 \mathrm{~kg} / \mathrm{cm}^{2}\right)$ para las placas y hormigón con una resistencia a la compresión $\mathrm{f}^{\prime} \mathrm{c}=350 \mathrm{~kg} / \mathrm{cm}^{2}$ como relleno. Las vigas son miembros de acero tipo "I" armados a base de placas soldadas. El material de las vigas es acero ASTM A36 $\left(\mathrm{Fy}=2500 \mathrm{~kg} / \mathrm{cm}^{2}\right)$. En los arriostramientos de pandeo restringido, el núcleo consiste en una placa plana hecha de acero ASTM A36 $\left(\mathrm{Fy}=2500 \mathrm{~kg} / \mathrm{cm}^{2}\right)$, mientras que el mecanismo de restricción del pandeo está compuesto por un tubo de acero, armado con dos perfiles tipo "C" (canales) doblados en frío, relleno con mortero.

La filosofía del Diseño Basado en Desempeño Sísmico (DBDS) fue empleada en los sistemas estructurales estudiados (PRMC y 
PAPR). Los Objetivos del Diseño por Desempeño (ODD) fueron relacionados a dos niveles sísmicos: el Sismo Considerado Máximo (MCE), el cual tiene una probabilidad de excedencia del 2\% en 50 años; y el Sismo de Diseño (DBE), defino como $2 / 3$ de la intensidad del nivel MCE. El enfoque de diseño propuesto para los PRMC y PAPR tiene dos objetivos de desempeño: (1) alcanzar el nivel de desempeño sísmico de Seguridad de Vida ante un nivel sísmico DBE; y (2) alcanzar el nivel de desempeño sísmico de Prevención del Colapso ante un nivel sísmico MCE (Herrera, 2005; Fahnestock, et al., 2006).

El edificio prototipo fue diseñado de acuerdo a la norma ASCE/SEI 7-10 (ASCE, 2010) y las especificaciones de AISC 341-10 (AISC, 2010b). Los efectos de la carga sísmica fueron estimados mediante un Análisis de Respuesta Espectral Modal (AREM), según lo establecido en la Sección 12.9 de ASCE/SEI 7-10 (ASCE, 2010).

El peso sísmico efectivo del edificio prototipo, W, fue estimado en 7280 ton (i.e., $W=7280$ ton). La estructura estudiada se asume es un edificio de oficinas típico; por consiguiente, por consiguiente, al mismo le corresponde una Categoría de Riesgo II, una Categoría de Diseño Sísmico D y un Factor de Importancia, IE, igual a 1.0 (i.e., $\mathrm{IE}=1.0)($ ASCE, 2010). Además, se asume que el edificio está ubicado sobre suelo rígido, el cual corresponde a una Clase de Sitio D (ASCE, 2010).

El Espectro de Respuesta Elástico de Diseño fue construido usando los parámetros de aceleración espectral en roca correspondiente a un nivel sísmico MCE, SS y S1. Actualmente, los valores de dichos parámetros, a nivel mundial, pueden ser obtenidos accediendo a mapas disponibles en el sitio web del Servicio Geológico de los Estados Unidos (United States Geological Survey, USGS, por su nombre y siglas en inglés). Los valores obtenidos para la ciudad de Guayaquil, Ecuador fueron de $1.90 \mathrm{~g}$ y $0.84 \mathrm{~g}$ para la aceleración de respuesta espectral MCE para períodos cortos, SS, y para un período igual a 1.0 segundo, S1, respectivamente. El Espectro de Respuesta Elástico de Diseño fue construido de acuerdo a lo indicado en la Sección 11.4.5 de ASCE/SEI 7-10 (ASCE, 2010). Los parámetros de diseño sísmico empleados, esto es, el factor de modificación de respuesta, $\mathrm{R}$, el factor de sobrerresistencia, $\Omega 0$, y el factor de amplificación de deflexiones, $\mathrm{Cd}$, se presentan en la tabla 1 (ASCE, 2010).
TABLA 1. PARÁMETROS DE DISEÑO SÍSMICO DE EDIFICIOS

\begin{tabular}{|lrrrr|}
\hline SISTEMA RESISTENTE A CARGAS SísMICAS (SRCS) & R & nO & CD \\
\hline Pórticos Resistentes a Momento Compuestos (PRMC) & 8 & 3 & 5.5 \\
\hline Pórticos con Arriostramientos de Pandeo Restringido (PAPR) & 8 & 2.5 & 5 \\
\hline
\end{tabular}

Para calcular las fuerzas internas de diseño de los diferentes elementos estructurales y para determinar los desplazamientos de la estructura, se desarrolló un modelo matemático tridimensional (3D) de elementos finitos, el cual se ilustra en la figura 6 , y se realizó un análisis lineal-elástico del edificio prototipo con la ayuda del programa SAP2000 (Habibullah \& Wilson, 1997).

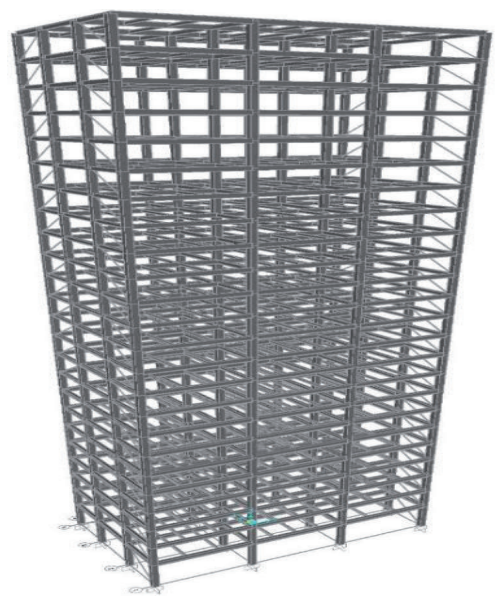

Figura 6. Modelo matemático tridimensional (3D) del edificio prototipo.

El modelamiento y análisis del edificio estuvieron basados en una serie de suposiciones, las cuales se resumen a continuación:

- Ambos sistemas estructurales (PRMC y PAPR) resisten cargas gravitacionales y cargas sísmicas;

- No se modeló el efecto de los elementos no estructurales (i.e., paredes);

- A nivel de la planta baja, las columnas fueron empotradas;

- Se asignó la condición de diafragma rígido a todos los nudos de una mismo nivel;

- No se consideró la flexibilidad de la zona de panel;

- Se asumieron conexiones totalmente restringidas para todos los pórticos del edificio;

- Se consideraron los efectos de torsión debido a una excentricidad accidental según lo recomendado por ASCE/SEI 7-10 (ASCE, 2010) en su Sección 12.8.4;

- No se tomó en cuenta la interacción suelo-estructura;

- Se consideraron criterios adicionales para el adecuado modelamiento de la 
rigidez axial y a flexión de las columnas CFT (Muhummud, 2003) y la rigidez axial de los arriostramientos de pandeo restringido (Hussain, et al., 2006).

Del análisis modal del edificio prototipo se obtuvo que el primer modo de vibración ocurre en forma de traslación en la dirección $\mathrm{X}$ con un período igual a 3.11 segundos (i.e., $\mathrm{T} 1 \mathrm{X}=3.27$ s.), mientras que el segundo modo de vibración corresponde a traslación en la dirección $\mathrm{Y}$ con un período igual a 2.90 segundos (i.e., $\mathrm{T} 1 \mathrm{Y}=3.06$ s.). La figura 7 presenta el Espectro de Respuesta Inelástico de Diseño empleado en el análisis elástico (AREM) del edificio prototipo; el espectro inelástico fue construido de acuerdo a lo indicado en la Sección 12.8.1.1 de ASCE/SEI 7-10 (ASCE, 2010). Como puede observarse en la figura 7, el valor del coeficiente de respuesta sísmica, Cs, obtenido para el análisis tanto en la dirección X (PRMC) como en la dirección Y (PRMC-PAPR) es el mínimo permitido por ASCE/SEI 7-10 (ASCE, 2010) (i.e., Cs = Cs,mín = 0.044). Por lo tanto, el cortante basal sísmico de diseño, Vdis, fue de 0.044W para el análisis en las dos direcciones.

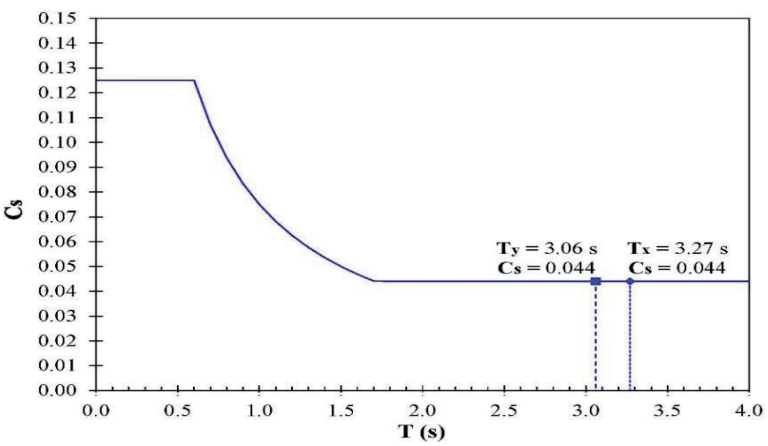

Figura 7. Espectro de respuesta inelástico de diseño.

Las dimensiones finales de los distintos elementos estructurales del edificio prototipo se indican en las tablas 2 y 3.

\begin{tabular}{|c|c|c|c|c|c|c|}
\hline \multicolumn{5}{|c|}{ PISO VIGAS PRINCIPALES } & \multicolumn{2}{|c|}{ VIGAS SECUNDARIAS } \\
\hline & \multicolumn{2}{|c|}{ Dirección X (PRMC) } & \multicolumn{2}{|c|}{ Dirección X (PRMC-PAPR) } & \multirow[b]{2}{*}{$\begin{array}{c}\text { Alas } \\
(\mathrm{mm})\end{array}$} & \multirow[b]{2}{*}{$\begin{array}{l}\text { Alma } \\
(\mathrm{mm})\end{array}$} \\
\hline & $\begin{array}{l}\text { Alas } \\
(\mathrm{mm})\end{array}$ & $\begin{array}{l}\text { Alma } \\
(\mathrm{mm})\end{array}$ & $\begin{array}{r}\text { Alas } \\
(\mathrm{mm})\end{array}$ & $\begin{array}{l}\text { Alma } \\
(\mathrm{mm})\end{array}$ & & \\
\hline 21 a 24 & $250 \times 18$ & $500 \times 8$ & $200 \times 16$ & $400 \times 8$ & $150 \times 8$ & $350 \times 6$ \\
\hline 17 a 20 & $250 \times 20$ & $500 \times 8$ & $200 \times 16$ & $400 \times 8$ & $150 \times 8$ & $350 \times 6$ \\
\hline 13 a 16 & $275 \times 20$ & $550 \times 10$ & $250 \times 18$ & $450 \times 8$ & $150 \times 8$ & $350 \times 6$ \\
\hline 9 a 12 & $275 \times 22$ & $550 \times 10$ & $250 \times 18$ & $450 \times 8$ & $150 \times 8$ & $350 \times 6$ \\
\hline 5 a 8 & $300 \times 22$ & $600 \times 10$ & $300 \times 20$ & $500 \times 8$ & $150 \times 8$ & $350 \times 6$ \\
\hline 1 a 4 & $300 \times 25$ & $600 \times 10$ & $300 \times 20$ & $500 \times 8$ & $150 \times 8$ & $350 \times 6$ \\
\hline
\end{tabular}

TABLA 3. DIMENSIONES DE LAS COLUMNAS CFT Y APR DEL EDIFICIO PROTOTIPO

PISO COLUMNAS (MM) ARRIOSTRAMIENTOS DE PANDEO RESTRINGIDO

\begin{tabular}{|c|c|c|c|}
\hline & & Núcleo de acero (mm) & Camisa de acero (mm) \\
\hline 21 a 24 & CFT 500x500x15 & PL60x15 & [] $200 \times 200 \times 3$ \\
\hline 17 a 20 & CFT 500x500x15 & PL80x15 & [] $200 \times 200 \times 3$ \\
\hline 13 a 16 & CFT 500x500x18 & PL100x15 & [] $200 \times 200 \times 4$ \\
\hline 9 a 12 & CFT 500x500x18 & PL100x18 & [] $200 \times 200 \times 4$ \\
\hline 5 a 8 & CFT $500 \times 500 \times 20$ & PL120x18 & [] $250 \times 250 \times 6$ \\
\hline $1 \mathrm{a} 4$ & CFT $500 \times 500 \times 20$ & PL120x20 & [] $250 \times 250 \times 6$ \\
\hline
\end{tabular}

La figura 8 ilustra la distribución de derivas de entrepiso del edificio prototipo en las direcciones X y Y. Se verificó que, con las dimensiones de los miembros (vigas, columnas CFT y APR) indicadas en las tablas 2 y 3 , la estructura no experimente valores de derivas de entrepiso mayores que la deriva de entrepiso admisible, esto es, un $2 \%$ de la altura de entrepiso ( 0.020 radianes), con el fin de dar cumplimiento al requerimiento establecido en ASCE/SEI 7-10 (ASCE, 2010) para los dos sistemas estructurales empleados (PRMC y PAPR). Las máximas derivas de entrepiso obtenidas fueron de 0.0181 radianes (en el entrepiso 10) y 0.0144 radianes (en el entrepiso 18) para el análisis en las direcciones X y Y, respectivamente. Por otra parte, los desplazamientos laterales elásticos amplificados (inelásticos) a nivel de cubierta fueron de $1111 \mathrm{~mm}$, correspondiente a una deriva global de 0.0141 radianes, y $939 \mathrm{~mm}$, correspondiente a una deriva global de 0.0119 radianes, para el análisis en las direcciones $\mathrm{X}$ y Y, respectivamente.

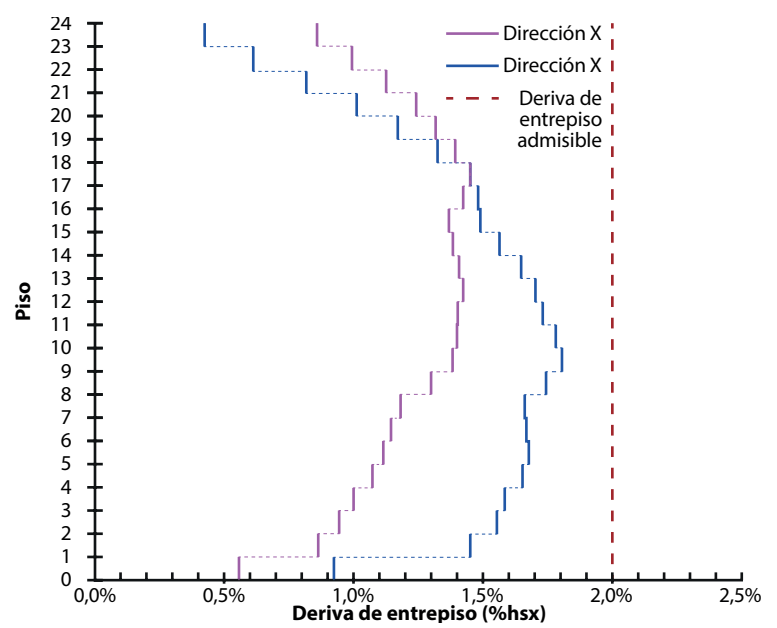

Figura 8. Derivas de entrepiso.

DISEÑO DE LOS PRMC DEL EDIFICIO PROTOTIPO Los requerimientos de derivas de entrepiso controlaron la selección del tamaño de los miembros estructurales de los PRMC (i.e., vigas y columnas 
CFT). Adicionalmente, los componentes de los PRMC fueron dimensionados de manera que se cumpliera, en todos los casos, el criterio de columna fuerte-viga débil, según lo indicado en AISC 341-10 (AISC, 2010b). Las columnas CFT y las vigas metálicas tipo "I" fueron diseñadas de acuerdo a las disposiciones de AISC 360-10 (AISC, 2010a) y AISC 341-10 (AISC, 2010b); los estados límites de los elementos más críticos fueron revisados de forma que no fueran excedidos. La capacidad a flexo-compresión de las columnas CFT fue determinada en base al Método de Distribución de Esfuerzos Plásticos como se describe en AISC 360- 10 (AISC, 2010a), y ésta a su vez fue comparada con la demanda obtenida. Las dimensiones de todos los elementos estructurales fueron adoptadas de manera que se satisfagan los límites de la relación ancho-espesor para miembros altamente dúctiles según lo requerido por AISC 341-10 (AISC, 2010b). Para los PRMC se empleó una conexión a momento viga-columna soldada con diafragmas interiores (placas de continuidad). En los PRMC del edificio prototipo se implementó la conexión a momento precalificada de vigas con sección reducida (CVSR). Las CVSR fueron diseñadas siguiendo el procedimiento descrito en el Capítulo 5 de AISC 358-10 (AISC, 2010c).

DISEÑO DE LOS PAPR DEL EDIFICO PROTOTIPO

En el caso de los PAPR, el diseño estuvo gobernado por resistencia. Tal como lo especifican los códigos, el núcleo de acero de los APR fue diseñado para resistir la carga axial total que se desarrolla en el arriostramiento debido a la acción sísmica. El área requerida de la sección transversal del núcleo de acero fue calculada en base al estado límite de fluencia en tensión y en compresión, según lo establecido en AISC 341-10 (AISC, 2010b). El tamaño del tubo de acero exterior de los APR fue seleccionado empleando el criterio propuesto por Watanabe et al. (1989). Este criterio establece que, para evitar la inestabilidad global del arriostramiento, la carga crítica de pandeo elástico (o carga de Euler) del tubo exterior debe ser al menos 1.5 veces la carga axial de fluencia del núcleo de acero del APR. Los miembros adyacentes al arriostramiento en los PARP (i.e., vigas, columnas y conexiones) fueron diseñados aplicando los principios del diseño por capacidad. Para los APR se empleó una conexión empernada estándar con placas gusset. Las conexiones de los APR fueron diseñadas en base al procedimiento desarrollado por Christopulos (2005). Por otra parte, las conexiones viga-columna de los PAPR, al igual que en los PRMC, son del tipo totalmente restringidas (o rígidas), es decir, se emplearon CVSR.

MODELO NO LINEAL DEL EDIFICIO PROTOTIPO

Con la finalidad de investigar el desempeño sísmico del edificio prototipo diseñado, dos modelos analíticos no lineales fueron desarrollados con la ayuda del programa OpenSees (McKenna et al., 2006). El primer modelo corresponde a un pórtico tipo en la dirección X (i.e., PRMC) mientras que el segundo modelo representa uno de los pórticos exteriores en la dirección Y (i.e., PAPR). Así, se evaluó el desempeño de estos dos sistemas estructurales para el diseño propuesto. La figura 9 muestra el modeloanalíticoparaelpórticotipoenladirección $\mathrm{X}$ (PRMC). Las columnas CFT fueron modeladas usando elementos viga-columna con secciones de fibras en los extremos y en una longitud igual a la dimensión de la columna (Herrera, 2005); el resto de la columna fue modelado mediante un elemento viga-columna con una sección elástica. Las vigas se modelaron usando elementos viga- columna con secciones elásticas y a sus nudos extremos les fueron asignados resortes rotacionales, los cuales toman en cuenta el deterioro de la resistencia y rigidez de la conexión viga-columna de acuerdo al modelo desarrollado por Lignos \& Krawinkler (2011). La rigidez de la zona de panel fue modelada de acuerdo a las recomendaciones de Fukumoto \& Morita (2005). La figura 10 ilustra el modelo analítico para el pórtico tipo en la dirección Y (PAPR). Este modelo es prácticamente el mismo que el empleado en el PRMC con la adición de los arriostramientos de pandeo restringido (APR). Los APR fueron modelados como elementos uniaxiales (truss), en la longitud correspondiente a la zona de fluencia (ver figura 3), cuya respuesta histerética fue calibrada de acuerdo a las recomendaciones de Santelices (2014), mientras que para las zonas de conexiones se usaron elementos viga-columna con comportamiento elástico.
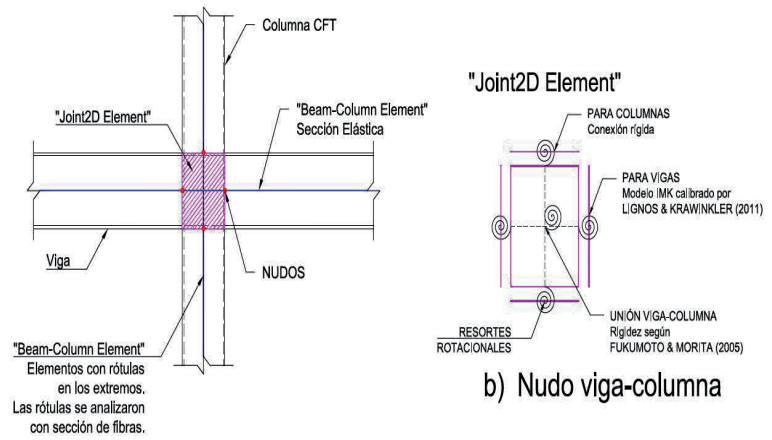

b) Nudo viga-columna

a) Elementos usados 


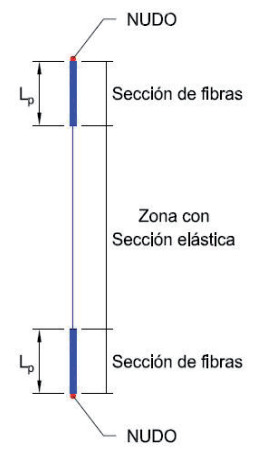

$L_{p}=B=$ Ancho de columna (HERRERA, 2005)

\section{c) Columna}

Figura 9. Modelo analítico no lineal OpenSees de los PRMC

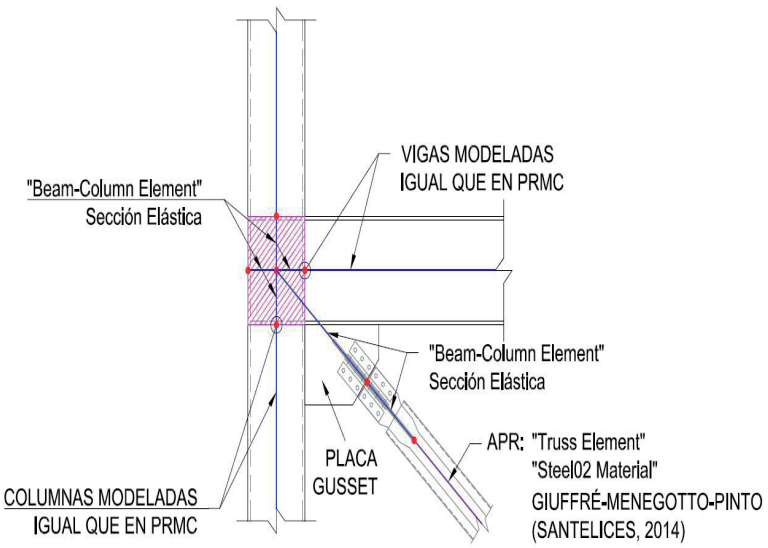

Figura 10. Modelo analítico no lineal OpenSees de los PAPR.

ANÁLISIS ESTÁTICO NO LINEAL

En cada pórtico se realizó análisis estáticos laterales (pushover). Las cargas laterales fueron distribuidas en los pórticos de acuerdo a los requerimientos de ASCE/SEI 7-10 (ASCE, 2010) y fueron aplicadas en las masas de ambos pórticos. La figura 11 presenta la relación entre el cortante basal normalizado (cortante basal dividido para el peso del edificio, $\mathrm{W}=7280$ ton) y la deriva total (desplazamiento de último piso dividido para la altura total del pórtico), $\theta$ total, para el PRMC. Los resultados de los análisis para el PRMC muestran que la primera fluencia ocurre cuando el cortante basal es $0.12 \mathrm{~W}$ $(\theta$ total $=1.10 \%)$. La sobrerresistencia máxima, $\Omega$ o, del PRMC es ligeramente menor a $4(0.172 \mathrm{~W})$ correspondiente a $\theta$ total de $2.75 \%$. La sobrerresistencia está definida como el cortante basal dividido para el cortante de diseño de ASCE/SEI 7-10 (ASCE, 2010). Por otra parte, la figura 12 muestra los resultados del análisis estático no lineal del PAPR, para el cual se tiene que la primera fluencia ocurre cuando el cortante basal es $0.11 \mathrm{~W}$ y $\theta$ total es $1.25 \%$. La sobrerresistencia máxima, $\Omega$ o, del PAPR es $4.32(0.190 \mathrm{~W})$ cuando $\theta$ total es $3.50 \%$.

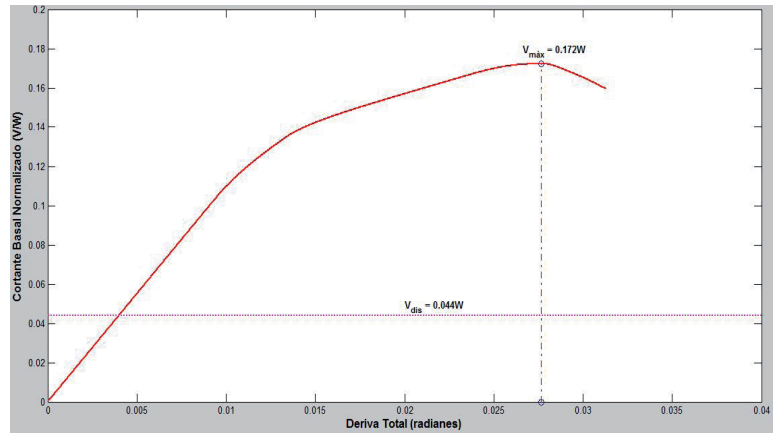

Figura 11. Resultados del Análisis Estático No Lineal (Pushover) Dirección X (PRMC).

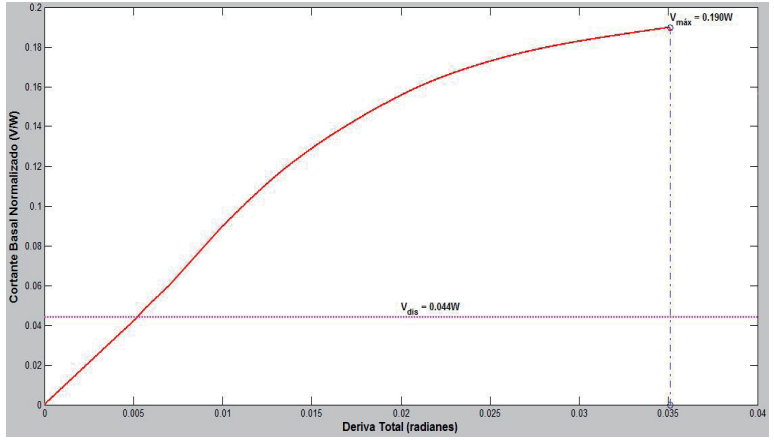

Figura 12. Resultados del Análisis Estático No Lineal (Pushover) Dirección Y (PAPR).

\section{ANÁLISIS DINÁMICO NO LINEAL}

Para la realización de análisis dinámicos nolineales, se utilizaron tres registros de aceleraciones, escalados a niveles de sismo de diseño (DBE) y de sismo considerado máximo (MCE). La figura 13 muestra el espectro de respuesta elástica de los registros empleados escalados al nivel DBE. Las figuras 13 y 14 presentan las magnitudes máximas de desplazamiento de piso, para cada registro escalado a los niveles DBE y MCE, del PRMC y el PAPR, respectivamente. La tabla 4 presenta los resultados de los análisis dinámicos de ambos sistemas (PRMC y PAPR) en lo concerniente a la deriva total y deriva de entrepiso, para los distintos registros de aceleraciones escalados a los niveles DBE y MCE.

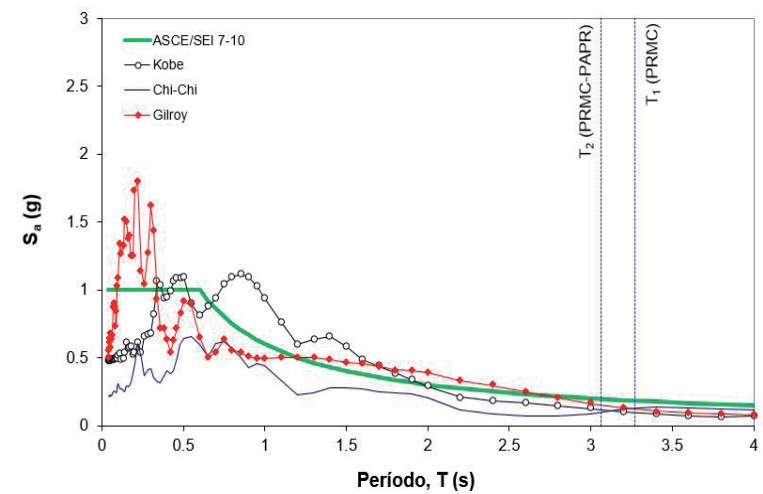

Figura 13. Espectros de Respuesta Elástica - Nivel DBE. 

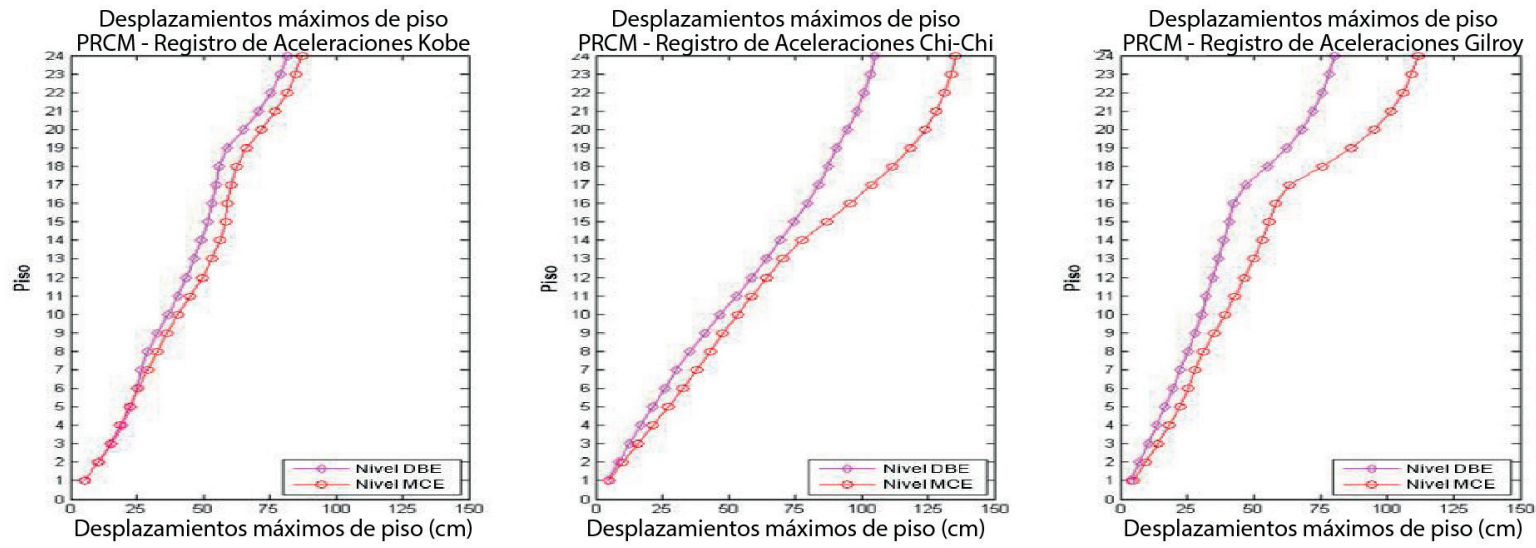

Figura 14. Resultados del Análisis Dinámico No Lineal - Desplazamientos máximos de piso PRMC
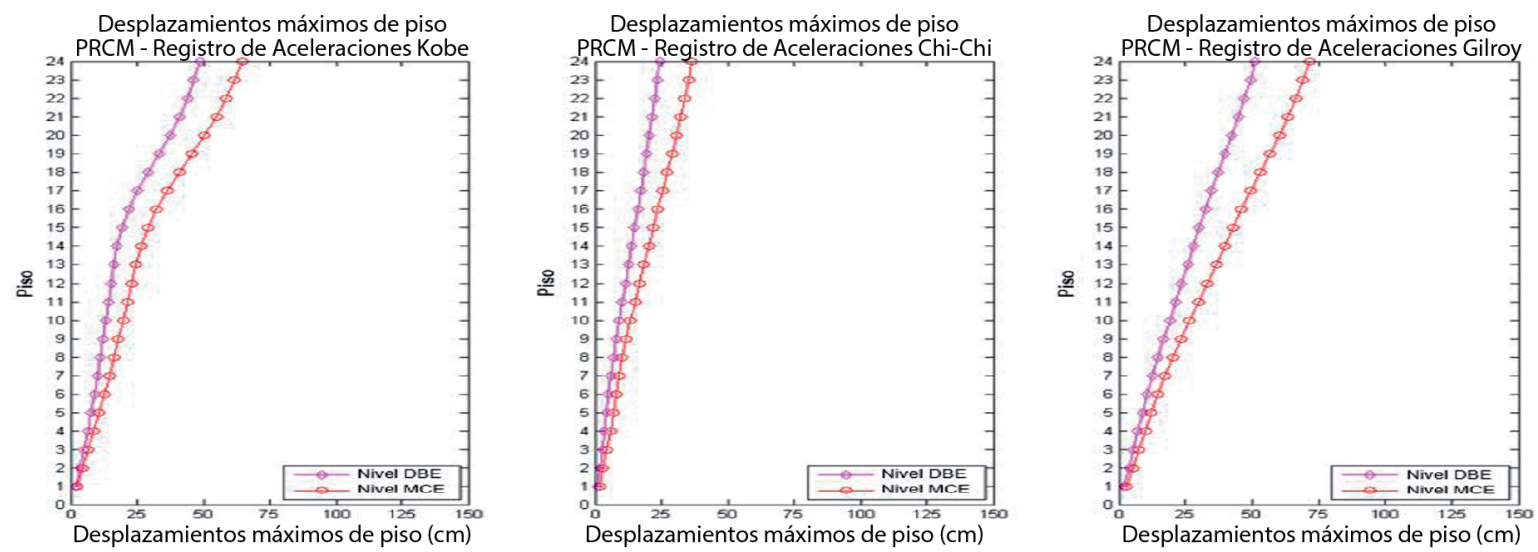

Figura 15. Resultados del Análisis Dinámico № Lineal - Desplazamientos máximos de piso PAPR

TABLA 4. RESULTADOS DEL ANÁLISIS DINÁMICO NO LINEAL - DERIVA TOTAL

\begin{tabular}{lrrrr}
\hline DERIVA & \multicolumn{1}{l}{ PRMC } & \multicolumn{3}{c|}{ PAPR } \\
TOTAL (\%) & Nivel DBE & Nivel MCE & Nivel DBE & Nivel MCE \\
\hline Kobe & 1.03 & 1.10 & 0.61 & 0.82 \\
\hline Chi-Chi & 1.33 & 1.71 & 0.31 & 0.46 \\
Gilroy & 1.02 & 1.41 & 0.79 & 0.95 \\
\hline
\end{tabular}

TABLA 5. RESULTADOS DEL ANÁLISIS DINÁMICO NO LINEAL - DERIVA DE ENTREPISO

\begin{tabular}{lrrrr} 
DERIVA & \multicolumn{1}{l}{ PRMC } & \multicolumn{3}{c}{ PAPR } \\
TOTAL (\%) & Nivel DBE & Nivel MCE & Nivel DBE & Nivel MCE \\
\hline Kobe & 2.34 & 2.89 & 1.28 & 1.78 \\
\hline Chi-Chi & 2.03 & 2.96 & 0.60 & 0.86 \\
\hline Gilroy & 2.49 & 3.80 & 1.15 & 1.50 \\
\hline
\end{tabular}

\section{RESUMEN, CONCLUSIONES Y RECOMENDACIONES}

Se presentó el análisis, diseño y la evaluación sísmica de un edificio prototipo de 24 pisos compuesto por PRMC y PAPR. El diseño del edificio fue realizado de acuerdo al procedimiento del diseño basado en desempeño sísmico y las disposiciones de ASCE/SEI 7-10 empleando un análisis elástico de respuesta espectral modal. La evaluación del desempeño sísmico del edificio prototipo diseñado fue realizada mediante análisis no lineales de dos tipos: (1) lateral estático (pushover); y (2) dinámico (tiempo-historia). Para los análisis no lineales se desarrollaron modelos analíticos de los sistemas estudiados (i.e., PRMC y PAPR) con la ayuda del programa OpenSees. En los análisis no lineales dinámicos se usaron tres registros de aceleraciones escalados a los niveles sísmicos DBE y MCE. Los resultados de la evaluación sísmica del edificio prototipo indican que ambos sistemas tienen un desempeño sísmico satisfactorio en términos de resistencia, ductilidad y capacidad de disipación de energía, y consecuentemente constituyen alternativas interesantes para la construcción de edificios altos y de mediana altura en nuestro país. Finalmente, trabajos futuros deberán abarcar el desarrollo de detalles constructivos de conexiones a momento viga-columna CFT y arriostramiento de pandeo restringido usando materiales y tecnología local con miras a llevar a cabo las fases experimentales del plan de investigación propuesto. 


\section{REFERENCIAS BIBLIOGRÁFICAS}

AISC (2010a). "Specification for Structural Steel Buildings". ANSI/AISC 341-10, American Institute of Steel Construction, Chicago, IL, United States.

AISC (2010b). "Seismic Provisions for Structural Steel Buildings”. ANSI/AISC 341-10, American Institute of Steel Construction, Chicago, IL, United States.

AISC (2010c). "Prequalified Connections for Special and Intermediate Steel Moment Frames for Seismic Applications”. ANSI/AISC 358-10, American Institute of Steel Construction, Chicago, IL, United States.

ASCE (2010). "Minimum Design Loads for Buildings and Other Structures". ASCE Standard ASCE/SEI 7-10, American Society of Civil Engineers, Reston, VA, United States, ISBN 978-0-7844- 1085-1.

Cassagne, A. (2009). “Estadodela Práctica del Diseño y Construcción de Edificios Existentes de Acero Resistentes a Momento y Recomendaciones para la Construcción de Edificios Nuevos de Acero en la Ciudad de Guayaquil”. Tesis de Grado, Facultad de Ingeniería en Ciencias de la Tierra, Escuela Superior Politécnica del Litoral, Guayaquil, Ecuador.

Christopulos, A.S. (2005). "Improved Seismic Performance of Buckling Restrained Braced Frames". M.S. Thesis, Department of Civil and Environmental Engineering, University of Washington, Seattle, WA, United States.

Crisafulli, F.J. (2013). "Diseño Sismorresistente de Construcciones de Acero, 3ra edición". Asociación Latinoamericana del Acero - Alacero.

Fahnestock, L.A., Ricles, J.M., R. Sause (2006). "Analytical and Experimental Studies of Earthquake- Resistant Buckling-Restrained Braced Frame Systems". ATLSS Reports, Paper 71, Bethlehem, PA, United States.

Fukumoto, T., Morita, K. (2005). "Elastoplastic Behavior of Panel Zone in Steel Beam-to-Concrete Filled Steel Tube Column Moment Connections". Journal of Structural Engineering, Vol. 131, No. 12, pp. 1841-1853.

Habibullah, A., Wilson, E. (1997). "SAP2000: Integrated Finite Element Analysis and Design of Structures". Computers and Structures Inc., Berkeley, CA, United States.

Herrera, R. (2005). "Seismic Behavior of Concrete Filled Tube Column-Wide-Flange Beam Frames". Ph.D. Dissertation, Department of Civil and
Environmental Engineering, Lehigh University, Bethlehem, PA, United States.

Hussain, S., Benschoten, P.V., Al Sarari, M., Lin, S. (2006). "Buckling Restrained Braced Frame (BRBF) Structures: Analysis, Design and Approval Issues". Proceedings of the 75th SEAOC Annual Convention, Long Beach, CA, United States.

Lignos, D.C., Krawinkler H. (2011). "Deterioration Modeling of Steel Components in Support of Collapse Prediction of Steel Moment Frames under Earthquake Loading”. Journal of Structural Engineering, Vol. 137, No. 11, pp. 1291-1302.

López, W.A., Sabelli, R. (2004). "Seismic Design of Buckling-Restrained Braced Frames". Steel Tips, Structural Steel Educational Council (SSEC), CA, United States.

McKenna, F., Fenves G.L., Filippou, F.C., Mazzoni, S. (2006). "Open System for Earthquake Engineering Simulation (OpenSees)". Pacific Earthquake Engineering Research Center (PEER), University of California, Berkeley, CA, United States. $<$ http://opensees.berkeley.edu>

Morino, S., Tsuda, K. (2003). "Design and Cons" truction of Concrete-Filled Steel Tube Column System in Japan”. Earthquake Engineering and Engineering Seismology, Vol. 4, No. 1, pp. 51-73.

Muhummud, T. (2003). "Seismic Behavior and Design of Composite SMRFs with Concrete Filled Steel Tube Columns and Steel Wide Flange Beams". Ph.D. Dissertation, Department of Civil and Environmental Engineering, Lehigh University, Bethlehem, PA, United States.

Sabelli, R., López, W.A. (2004). "Design of BucklingRestrained Braced Frames". Proceedings of the North American Steel Construction Conference (NASCC), Long Beach, CA, United States.

Santelices, K. (2014). "Aplicación de Arriostramientos de Pandeo Restringido a una Estructura Industrial". Memoria para optar al título de Ingeniero Civil, Facultad de Ciencias Físicas y Matemáticas, Universidad de Chile, Santiago, Chile.

Watanabe, A., Hitomi, Y., Saeki, E., Wada, A., Fujimoto, M. (1988). "Properties of Brace Encased in Buckling-Restraining Concrete and Steel Tube". Proceedings of the 9th World Conference on Earthquake Engineering, Vol. IV, pp. 719-724, August 2-9, Tokyo-Kyoto, Japan. 